\title{
Comparison between Forceps, Single Blade Forceps and Manual Extraction of Fetal Head in Elective Caesarean Section: A Randomized Control Trial*
}

\section{-Forceps Delivery in Cesarean Section}

\author{
Ahmed Sherif Abdel Hamid Abdel Wahab"\#, Ashraf Sobhy Aboulouz ${ }^{2}$ \\ ${ }^{1}$ Lecturer/Obstetrics \& Gynecology, Department of Obstetrics \& Gynecology, Ain-Shams University, Cairo, Egypt \\ ${ }^{2}$ Lecturer/Obstetrics \& Gynecology, Department of Obstetrics \& Gynecology, October 6th University, Cairo, Egypt \\ Email: "ahmedgyna@yahoo.com
}

How to cite this paper: Abdel Hamid, A.S. and Aboulouz, A.S. (2018) Comparison between Forceps, Single Blade Forceps and Manual Extraction of Fetal Head in Elective Caesarean Section: A Randomized Control Trial-Forceps Delivery in Cesarean Section. Open Journal of Obstetrics and Gynecology, 8, 31-38.

https://doi.org/10.4236/ojog.2018.81004

Received: December 8, 2017

Accepted: January 9, 2018

Published: January 12, 2018

Copyright ( $) 2018$ by authors and Scientific Research Publishing Inc. This work is licensed under the Creative Commons Attribution International License (CC BY 4.0).

http://creativecommons.org/licenses/by/4.0/ (c) (i) Open Access

\begin{abstract}
Objective: The fundal pressure exerted by the assistant to deliver fetal head is often painful to the patient. This study assesses the use of double blade forceps in delivery of fetal head at time of elective Cesarean Section (CS). Methods: A prospective single-blinded randomized controlled trial was conducted among 150 women with repeat elective CS at 3 different hospitals. Women were classified into 3 groups (each 50 women). Forceps group: A double blade of forceps was used without fundal pressure. Single blade group: single blade of forceps was used assisted by fundal pressure. Manual group: manual extraction was used assisted by fundal pressure. The outcome of study were; Pain expectation score, pain score during delivery of head, unintended uterine extension, uterine vessels injury and need for additional stitches. The collected data were statistically analyzed using SPSS version 20 . Results: High Statistically significant difference in pain score during delivery of head in favor of forceps group $(P=0.001)$. No differences were found among 3 groups as regarding pain expectation, uterine extension, uterine vessel injury and in need of haemostatic stitches $(P>0.05)$. Conclusion: Use of double blade forceps is less painful for the patients during delivery of head in CS.
\end{abstract}

\section{Keywords}

Forceps Extraction, Fundal Pressure, Manual Extraction, Pain

*Trial registration: PAN African clinical Trial registry ID Number: pactr 201710002607341 


\section{Introduction}

Cesarean delivery is the most common and major obstetric operative procedure worldwide and cesarean rate has been continuously increased [1].

In the United States most primary cesarean deliveries are performed for the indication of dystocia in labor. In these cases the fetal head is well engaged and the lower uterine segment has been thinned by the forces of labor. If the fetal head is not deeply engaged in the maternal pelvis, it is usually a simple maneuver to make a transverse lower uterine segment incision and lift the fetal head to the level of the incision and effect delivery [2].

Most elective cesarean deliveries are performed under regional anesthetic (whether epidural or spinal) for reason of patient safety and satisfaction [3].

Several methods have been described for the delivery of the fetal head at the time of elective cesarean delivery. The most common delivery is simple manual delivery. The fundal pressure exerted by the surgeon and the assistant in an effort to deliver an unengaged fetal vertex through a thick lower uterine segment is often perceived as uncomfortable, even painful, by the patient [4].

Therefore we will conduct a study to compare 3 methods of delivery of the fetal head (forceps-assisted using either single blade or double blade versus manual delivery) at time of elective cesarean section as regards patient expectation of pain and pain scores, unintended extension of the uterine incision, uterine vessels injury and need of additional stitches.

\section{Methods}

This prospective multicentre randomized control study was carried out at 3 different hospitals during the period from September till December 2017. The ethics committee of the 3 Hospitals approved the study. The study included women aged between 18 and 45 years, with history of previous CS delivery with spinal anesthesia. All women had a scheduled delivery at 38 week or 39 week. Excluded from the study were the women with previous CS delivery with general anesthesia, Women who cannot remember the pain felt during their last CS, preterm pregnancy, women in labor, multiple pregnancies, fetal malpresentation (non cephalic presentation) and fetal congenital malformation.

All women were informed about the purpose of the trial, the operation modalities, and their benefits as well as risks. All women gave their informed consent, after which detailed history was taken, clinical examination, obstetric ultrasonography was performed to calculate gestational age, fetal growth, amniotic fluid and to exclude fetal congenital malformation.

Since there was currently no adequate information regarding the expected difference in the outcome measures between the three groups under investigation, the current exploratory study would target an effect size ( $\mathrm{n}=50$ per group) that would be clinically relevant.

A total of 150 women who met the inclusion criteria were recruited to the study. The women were randomly allocated into three groups of 50 . The alloca- 
tion sequence was generated by simple randomization using a randomization table created by a computer software program. The allocation sequence was concealed by sealed opaque envelopes.

Participants were asked to mark their expectation of pain before the procedure depending to their previous experience in previous CS and the pain felt during fundal pressure. A standard $10 \mathrm{~cm}$ VAS was used to measure pain [5], in which $0 \mathrm{~cm}$ corresponded to "no pain" and $10 \mathrm{~cm}$ corresponded to "the worst pain ever".

After having the routine preoperative lab the women had their CS. All the women had spinal anesthesia.

The surgical steps up to the point of fetal extraction were accomplished in a standard fashion. Briefly the operation is initiated with Pfannenstiel skin incision performed with scalpel. The subcutaneous incision and opening were performed with the electrocautery; a transverse opening of the rectus sheath was made with the scalpel and extended laterally with scissors; the peritoneum was opened by scissors, downward dissection of the bladder downward. Opening of the lower uterine segment was performed by scalpel in the traditional $\mathrm{C}$ shape incision then incision of fetal membranes was performed to reach fetal head.

F group: 50 women that we used double blades forceps for head extraction: Once the uterine incision has been performed and the membranes are ruptured, the surgeon confirmed the transverse position of the head. The fixed posterior blade was placed first; the surgeon placed one hand under the head at temporal bone behind the posterior ear and slided the curved blade between the surgeon fingers, moving the fetal head into position. The anterior blade then was then applied directly to the correct position in front of the anterior ear. Traction was then applied, without rotation, along the longitudinal axis of the mother the delivery of the fetus, placenta and membranes. Closure of uterus by Vicryl 1 - 0 in continuous 2 layers manner. Any unintended extension, uterine vessels injury, additional stitches were recorded. The rest of procedure was as following; placing intraperitoneal drain when needed, closure of parital peritoneum and then closure of abdomen in layers.

S Group: $\mathbf{5 0}$ women that we used only one blade forceps for head extraction: The fixed posterior blade is placed first; the surgeon placed one hand under the head at temporal bone behind the posterior ear and slided the curved blade between the surgeon fingers, moving the fetal head into position then asking the assistant to perform fundal pressure to facilitate delivery of head. The rest of procedure continues as standard way we described.

M Group: 50 women that we delivered head by manual extraction: The surgeon's dominant hand was placed into the lower uterine segment and his fingers cupped the fetal head. Firm, gentle traction was used to elevate the fetal head towards the incision. The fetal head might be then rotated to an occipitoanterior position and delivered through the uterine incision with the assistance of fundal pressure. The rest of procedure continues as standard way we de- 
scribed.

The women were asked to concentrate on the amount of discomfort or pain that they experienced as the baby is being delivered especially of the fundal pressure if exerted. A standard $10 \mathrm{~cm}$ VAS was used to measure pain [5], in which $0 \mathrm{~cm}$ corresponded to "no pain" and $10 \mathrm{~cm}$ corresponded to "the worst pain ever".

During the operation, the unintended uterine extension, uterine vessels injury, need for additional stitches in closing uterine incision were recorded to be compared in different techniques.

The birth weight, Apgar score at 1 minute and 5 minutes were recorded for all deliveries.

The collected data was revised, coded, tabulated and introduced to a PC using Statistical package for Social Science (IBM SPSS Statistics for Windows, Version 20.0. Armonk, NY: IBM Corp). Quantitative variables are expressed as mean and SD. Qualitative variables are expressed as frequencies and percents. Student $t$ test was used to compare a continuous variable between three study groups. ANOVA with Bonferroni post hoc test was used to compare a continuous variable between more than three study groups. Chi square and Fisher's exact tests were used to examine the relationship between Categorical variables. P-value $<0.05$ was considered statistically significant .Data were collected, tabulated,

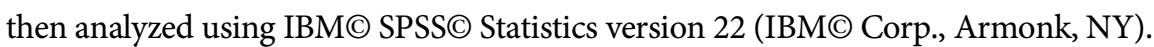

\section{Results}

A total of 150 pregnant women were enrolled to the study (Figure 1). They were randomized into three groups; 50 women in each group. The baseline demographic characteristics were similar between both three groups (Table 1). There were no statistical significant differences between studied groups as regard maternal age, gestational age, Body mass index or Parity $(\mathrm{P}>0.05)$.

There was no statistical significant difference regarding the expectation of pain depending on previous experience of last CS. The women were asked to concentrate on the amount of pain that they experienced as the baby is being delivered especially of the fundal pressure if exerted. There was a high statistical significant difference in pain scores where less pain was found in double blade forceps group in comparison to single blade group or manual extraction group $(\mathrm{P}=0.001)($ Table 2$)$.

There were no statistical significant differences between studied groups as regard unintended uterine extensions, uterine vessels injury or need of additional stitches. As regard neonatal outcome; birth weight, APGAR scores at 1 minute and 5 minutes were not different between studied groups (Table 2).

There were no cases of fetal birth injuries in any of the groups.

\section{Discussion}

The present study found no significant differences between the three groups as 


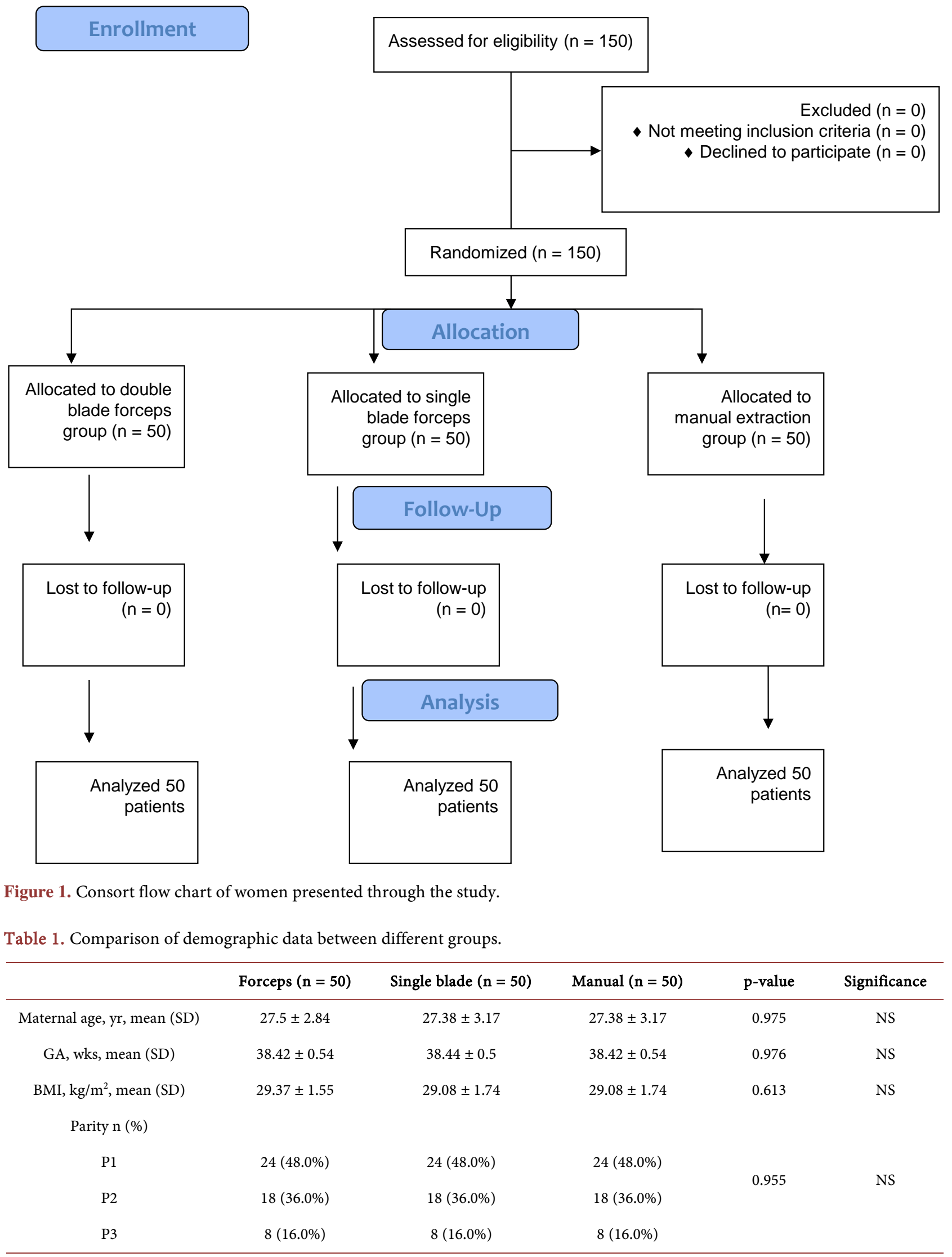

GA = Gestational Age, BMI = Body Mass Index, $\mathrm{P}=$ Parity, NS = Non Significant. 
Table 2. Comparison of outcome variables data between different groups.

\begin{tabular}{|c|c|c|c|c|c|c|}
\hline & & Forceps $(n=50)$ & Single blade $(n=50)$ & Manual $(n=50)$ & p-value & Significance \\
\hline \multicolumn{2}{|c|}{ Expectation of pain, mean (SD) } & $6.2 \pm 1.7$ & $5.6 \pm 1.8$ & $5.5 \pm 1.7$ & 0.09 & NS \\
\hline \multicolumn{2}{|c|}{ Pain during fundal pressure, mean (SD) } & $4.5 \pm 2.1$ & $5.8 \pm 2.2$ & $6.0 \pm 1.5$ & 0.001 & HS \\
\hline \multirow{3}{*}{ Uterine extension } & Negative & $47(94.0 \%)$ & $45(90.0 \%)$ & $43(86.0 \%)$ & \multirow{3}{*}{0.394} & \multirow{3}{*}{ NS } \\
\hline & & & & & & \\
\hline & Positive & $3(6.0 \%)$ & $5(10.0 \%)$ & $7(14.0 \%)$ & & \\
\hline \multirow{3}{*}{ Uterine vessels injury } & Negative & 48 (96.0\%) & 46 (92.0\%) & $44(88.0 \%)$ & \multirow{3}{*}{0.466} & \multirow{3}{*}{ NS } \\
\hline & & & & & & \\
\hline & Positive & $2(4.0 \%)$ & $4(8.0 \%)$ & $6(12.0 \%)$ & & \\
\hline \multirow{3}{*}{ Additional stitches needed } & Negative & $42(84.0 \%)$ & $35(70.0 \%)$ & $35(70.0 \%)$ & \multirow{3}{*}{0.178} & \multirow{3}{*}{ NS } \\
\hline & & & & & & \\
\hline & Positive & $8(16.0 \%)$ & $15(30.0 \%)$ & $15(30.0 \%)$ & & \\
\hline \multicolumn{2}{|l|}{ Birth Weight } & $3.51 \pm 0.33$ & $3.54 \pm 0.25$ & $3.51 \pm 0.26$ & 0.802 & NS \\
\hline \multicolumn{2}{|c|}{ APGAR at $1 \mathrm{~min} \leq 7$} & $7(14)$ & $6(12)$ & $6(12)$ & 0.942 & NS \\
\hline \multicolumn{2}{|c|}{ APGAR at $5 \mathrm{~min} \leq 7$, } & $1(2)$ & $1(2)$ & $1(2)$ & 1.00 & NS \\
\hline
\end{tabular}

HS= Highly Significant, NS=Non Significant.

regards participants' age, gestational age, BMI and parity. Pain felt during delivery of the baby was significantly less in the first group in which delivery was done by application of two blades of forceps. However, there were no significant differences between the groups as regards expectation of pain.

The wide use of spinal anesthesia now in performing CS allows the patient to be aware of all steps in CS. Our results show that the patient remembered the pain they experienced in the previous CS. The expectation of pain was not different among three groups.

The fundal pressure not only causes pain and discomfort but also it may have other effects. Very few studies have been concerned with use of fundal pressure in CS. In study of Kurtay et al. [6] they studied the Effect of Uterine Fundal Pressure on Maternal intraocular Pressure in Cesarean Delivery. They concluded that fundal pressure may significantly increase the intraocular pressure.

Another study of Kim and Ryu [7] they studied the effect of fundal pressure at Caesarean section on maternal hemodynamics of 20 women. They studied brachial arterial blood pressure, heart rate and cardiac output during exertion of fundal. They found that applying fundal pressure produces significant hemodynamic effects in the form of significant decrease in heart rate, cardiac output, blood pressure and mean systolic aortic flow time. They did not observe any clinical effect on mothers and babies.

There was no statistical significant difference between 2 groups as regard unintended uterine expansion, uterine vessel injury or need of additional stitches. This reflects that neither technique have an advantage over the other one especially when surgeons are well trained with different methods of delivery of head. Also there was no difference regarding neonatal outcome and APGAR scores. 
This reflects that using the forceps is safe in delivery of head in CS.

To the best of our knowledge, this is the first study to study the pain and discomfort felt by the patient during exerting fundal pressure in Cesarean section.

Potential limitations of the present study included the relative small number of participants in each group and the lack of sample size justification as no similar studies were published.

The standardized surgical steps, strictly criteria of selecting the women and no drop out of the participants were our strong points.

The results of the above study showed that since the pain felt by the women is significantly lower, so the surgeons should be encouraged to avoid fundal pressure by use of double blade of forceps to avoid patient discomfort and increase patient satisfaction.

Further research is needed with larger number of participants to study the blood loss with the use of forceps in CS.

\section{Conclusion}

Compared with fundal pressure exerted with manual extraction of fetal head or use of single blade forceps, the use of double blade forceps resulted in significant less pain felt by the patient during delivery of fetus. The study recommends wider use of forceps delivery and avoidance of fundal pressure in CS.

\section{Author Contribution}

A Sherif: Project development, Patient Recruitment, data collection and manuscript writing.

A Sobhy: Patient Recruitment, data collection and manuscript revision.

\section{Acknowledgements}

The authors would like to thank the staff and residents of 3 hospitals for their contribution in the study.

\section{Conflict of Interest}

The authors declare that there is no conflict of interest associated with this manuscript.

\section{Synopsis}

Forceps extraction of fetal head avoids the pain of the patient resulting from assisted fundal pressure during manual extraction of fetal head in Cesarean Section.

Type of Article: Clinical article.

\section{References}

[1] Cunningham, F.C., Kenneth, J. and Steven, L. (2010) Cesarean Delivery and PERIPARTum. Williams Obstetrics, 23, 544-563. 
[2] Warenski, J.C. (1981) A Technique to Facilitate Delivery of the High-Floating Head at Cesarean Delivery. American Journal of Obstetrics \& Gynecology, 139, 625-627. https://doi.org/10.1016/0002-9378(81)90473-7

[3] Depp, R. (1996) Cesarean Delivery. In: Gabbe, S.G., Niebyl, J.R. and Simpson, J.L., Eds., Obstetrics: Normal and Problem Pregnancies. 3rd Edition, Churchill Living-Stone, New York, 578.

[4] Barton, L.G., Caldwell, W.E. and Studdiford, W.E. (1928) The New Obstetric Forceps. American Journal of Obstetrics \& Gynecology, 15, 16-26. https://doi.org/10.1016/S0002-9378(15)32676-4

[5] Portenoy, R.K. and Tanner, R.M. (Eds.) (1996) Pain Management: Theory and Practice. Oxford University Press, Oxford.

[6] Kurtay, A., Ozayar, E., Gulec, H., Yildiz, G., Turkyilmaz, E., Yildiz, Z. and Horasanli, E. (2017) Effect of Uterine Fundal Pressure on Maternal intraocular Pressure in Cesarean Delivery: Comparison of Regional and General Anesthesia. Journal of Glaucoma, 26, 708-711. https://doi.org/10.1097/IJG.0000000000000687

[7] Kim, T.Y. and Ryu, D.H. (2006) The Effect of Fundal Pressure at Caesarean Section on Maternal Haemodynamics. Anaesthesia, 61, 434-438.

https://doi.org/10.1111/j.1365-2044.2006.04612.x 